This item was submitted to Loughborough's Research Repository by the author.

Items in Figshare are protected by copyright, with all rights reserved, unless otherwise indicated.

\title{
The effect of running velocity on footstrike angle - a curve-clustering approach
}

PLEASE CITE THE PUBLISHED VERSION

http://dx.doi.org/10.1016/j.gaitpost.2014.08.004

PUBLISHER

(C) Elsevier B.V.

VERSION

AM (Accepted Manuscript)

\section{PUBLISHER STATEMENT}

This work is made available according to the conditions of the Creative Commons Attribution-NonCommercialNoDerivatives 4.0 International (CC BY-NC-ND 4.0) licence. Full details of this licence are available at: https://creativecommons.org/licenses/by-nc-nd/4.0/

\section{LICENCE}

CC BY-NC-ND 4.0

\section{REPOSITORY RECORD}

Forrester, Stephanie E., and J. Townend. 2019. "The Effect of Running Velocity on Footstrike Angle - a Curveclustering Approach". figshare. https://hdl.handle.net/2134/16690. 


\title{
The Effect of Running Velocity on Footstrike Angle - A Curve-Clustering Approach
}

\begin{abstract}
S.E. Forrester and J. Townend
Wolfson School of Mechanical and Manufacturing Engineering, Loughborough University, Loughborough, LE11 3TU, UK
\end{abstract}

Keywords: $\quad$ footstrike pattern, footstrike angle, running velocity, curve-cluster analysis

Address for correspondence:

Dr Stephanie Forrester, Wolfson School of Mechanical and Manufacturing Engineering, Loughborough University, Loughborough LE11 3TU, UK. Email: s.forrester@lboro.ac.uk. Tel: $+44(0) 1509564824$

Received date: $15-8-2013$

Revised date: 10-6-2014

Accepted date: 8-8-2014

DOI: http://dx.doi.org/doi:10.1016/j.gaitpost.2014.08.004 


\section{ABSTRACT}

Despite a large number of studies that have considered footstrike pattern, relatively little is known about how runners alter their footstrike pattern with running velocity. The purpose of this study was to determine how footstrike pattern, defined by footstrike angle (FSA), is affected by running velocity in recreational athletes. One hundred and two recreational athletes ran on a treadmill at up to ten set velocities ranging from $2.2-6.1 \mathrm{~m} \cdot \mathrm{s}^{-1}$. Footstrike angle (positive rearfoot strike, negative forefoot strike), as well as stride frequency, normalised stride length, ground contact time and duty factor, were obtained from sagittal plane high speed video captured at $240 \mathrm{~Hz}$. A probabilistic curve-clustering method was applied to the FSA data of all participants. The curveclustering analysis identified three distinct and approximately equally sized groups of behaviour: (1) small / negative FSA throughout; (2) large positive FSA at low velocities $\left(\leq 4 \mathrm{~m} \cdot \mathrm{s}^{-1}\right)$ transitioning to a smaller FSA at higher velocities $\left(\geq 5 \mathrm{~m} \cdot \mathrm{s}^{-1}\right)$; (3) large positive FSA throughout. As expected, stride frequency was higher, while normalised stride length, ground contact time and duty factor were all lower for Cluster 1 compared to Cluster 3 across all velocities; Cluster 2 typically displayed intermediate values. These three clusters of FSA - velocity behaviour, and in particular the two differing trends observed in runners with a large positive FSAs at lower velocities, can provide a novel and relevant means of grouping athletes for further assessment of their running biomechanics. 


\subsection{INTRODUCTION}

There has been growing interest in footstrike patterns (FSP) during running, particularly given recent suggestions of a link to injury [1-4]. Footstrike patterns have been most commonly considered for middle- and long-distance runners and qualified by discrete classification, i.e. rearfoot strike (RFS) where the heel contacts the ground first; midfoot strike (MFS) where both the heel and ball of the foot land at approximately the same time; and forefoot strike (FFS) where the ball of the foot contacts the ground first, e.g. [5]. Studies on long-distance runners of varying ability have reported that 75-99\% RFS, 0-24\% MFS, and the remaining 0-2\% FFS [5-8]. Thus, the majority of long-distance runners RFS, while differences in the prevalence of MFS are most likely due to the subjective nature of the discrete classification method and, in particular, the difficulty in distinguishing between MFS and FFS (e.g. see Figure 1 in [5]) as well as differences in the ability and type of runners studied.

Running velocity has been suggested as a factor affecting FSP. Hasegawa et al. [5] found evidence for a trend towards reduced RFS and increased MFS in faster half-marathon runners. Hayes and Caplan [9] found a more even distribution of discrete FSP classifications in highcalibre middle-distance runners (800m and $1500 \mathrm{~m}$ track races) with $27 \%$ RFS, $42 \%$ MFS and $31 \%$ FFS. Although these studies have suggested a trend away from RFS as running velocity increases, they are unable to differentiate between the contributions of running velocity, the runners themselves (i.e. are faster runners more likely to MFS regardless of velocity?) and footwear.

Both Keller et al. [10] and Nigg et al. [11] were able to isolate the effects of running velocity by measuring FSP [10] or sole angle [11] for a group of runners in consistent footwear over a range of velocities $\left(3-7 \mathrm{~m} \cdot \mathrm{s}^{-1}\right)$. Both studies found that runners who predominantly RFS at running velocities below $5 \mathrm{~m} \cdot \mathrm{s}^{-1}$, shifted towards a more MFS or FFS at velocities above $\sim 5-6 \mathrm{~m} \cdot \mathrm{s}^{-1}$. This 
was suggested to enable them to cope with the higher collision forces associated with the higher velocities [11]. These studies provide stronger evidence that a runner's FSP is dependent on running velocity; however, both had limitations. Keller et al. [10] estimated FSP from $30 \mathrm{~Hz}$ video which may have been too low for reliable classification. Nigg et al. [11] presented only group mean and standard deviation sole angles, thus individual differences could not be assessed despite the standard deviation increasing by almost a factor of two between the lowest and highest velocities (from $4.7^{\circ}$ at $3 \mathrm{~m} \cdot \mathrm{s}^{-1}$ to $7.9^{\circ}$ at $6 \mathrm{~m} \cdot \mathrm{s}^{-1}$ ). A further limitation of the discrete FSP classification method, i.e. RFS, MFS or FFS, is the poor resolution meaning that subtle changes in FSP with running velocity are missed. Indeed, considering the injury statistics for long-distance runners (between 19\% and 79\% attain at least one injury per year $[1,12]$ ) and the suggested link to FSP [1-4], is it really beneficial to group $>75 \%$ of runners into a single category (RFS) in our search for greater understanding?

Despite the widespread use of discrete classification, in reality FSP is a continuum from extreme RFS to extreme FFS. Indeed, FSP was first quantified by the continuous measure of Strike Index [13]; the centre of pressure location at touchdown measured as a percentage along the long axis of the foot from heel to toe, i.e. 0-33\% indicates RFS, 34-67\% MFS, and 68-100\% FFS. However, force platforms have their limitations: they are difficult to use in natural running environments; targeting is a recognised issue; and centre of pressure values tend to be inaccurate until the ground reaction forces exceed a threshold value $[14,15]$. More recently, footstrike angle (FSA) has been shown to be an acceptable continuum measure of FSP [16] that overcomes the limitations associated with Strike Index and discrete classification methods. Footstrike angle is the sagittal plane foot angle measured with respect to the ground at the instant of touchdown and can be determined from 3D motion capture [16] or high speed video [11]. 
Despite the large number of studies that have considered FSP, very few have considered the effects of running velocity on an individual's FSP. Hence, our current knowledge of how individuals adapt their FSP across the range of velocities representative of slow jogging through to sprinting remains largely unknown. Such information may be relevant in our search for understanding of FSP and injury. Therefore, the purpose of this study was to determine how FSP (defined by FSA) is affected by running velocity in recreational athletes. It was hypothesized that the majority of individuals would have large positive FSAs at lower velocities (i.e. RFS) and that FSA would reduce as velocity increased (i.e. move towards MFS / FFS) particularly above $5 \mathrm{~m} \cdot \mathrm{s}^{-1}$.

\subsection{METHODS}

\subsection{Participants}

One hundred and two participants $(67$ males $23.3 \pm 5.1$ years, $1.80 \pm 0.07 \mathrm{~m}, 76.8 \pm 10.7 \mathrm{~kg}$ and 35 females $23.5 \pm 6.6$ years, $1.68 \pm 0.07 \mathrm{~m}, 61.7 \pm 5.6 \mathrm{~kg}$ ) provided voluntary written informed consent to participate in the study which was approved by Loughborough University Ethical Advisory Board. All participants took part in physical activity at a recreational level for a minimum of one hour a week, with an average participation of $7.7 \pm 4.2$ hours per week. The physical activities carried out by the participants were varied and included: running (track, road and trail), badminton, basketball, soccer, hockey, netball, rugby union, squash, tennis and triathlon. All participants had been free from running related injuries in the three months prior to testing.

\subsection{Protocol}

All running trials were performed on a treadmill (Mercury LT med, HP Cosmos, NussdorfTraunstein, Germany) with footstrike characteristics measured from sagittal plane high speed video (Casio Exilim EX-FH100, Casio America, Inc., Dover, NJ, USA) recording at $240 \mathrm{~Hz}$ 
(shutter speed: $1 / 250$ th second; spatial resolution: $448 \times 336$ pixels). The camera was positioned 3 $m$ from the treadmill which ensured that any out of plane foot movement would give only small errors in FSA, estimated at $<1^{\circ}[17,18]$.

Following a short warm up, of self-selected speed and duration, participants were asked to run at ten incrementally increasing velocities from 2.2 to $6.1 \mathrm{~m} \cdot \mathrm{s}^{-1}$ with the gradient maintained at $0 \%$ throughout. The lowest velocity was just above the walk-run transition [19] while the highest velocity was limited by the treadmill maximum. The participants were required to run for 60 seconds at each velocity and were allowed to stop as soon as they felt they no longer had the ability to run at the set speed. Up to two minutes rest was provided between velocities to minimise the effects of fatigue. High speed video was captured for five seconds starting 30 seconds into each running velocity trial. Due to the uncertainty in the highest velocity that would be reached by each participant and to allow the participants to become accustomed to the highest treadmill velocities, all started at the lowest velocity and progressed towards the highest velocity.

\subsection{Data analysis}

The high speed video footage was analysed using Image-Pro Plus 6.0 (Media Cybernetics Inc., Rockville, MD, USA) to determine the following five variables: footstrike angle; stride frequency; stride length (normalised to body height), ground contact time and duty factor (ratio of ground contact time to stride time). Footstrike angle was determined according to the method described by Altman and Davis [16] with the aid of markers placed on the rear and forefoot of the participant's shoe. Moderate to large positive angles represented a rearfoot strike, angles close to $0^{\circ}$ a mid-foot strike and moderate to large negative angles a forefoot strike. Three footstrikes per participant and velocity were analysed with the mean used in further analysis. In addition to measuring FSA, discrete FSP classification was conducted based on the definitions for RFS, MFS and FFS given in the Introduction, i.e. rearfoot strike (RFS) where the heel contacts the ground 
first; midfoot strike (MFS) where both the heel and ball of the foot land at approximately the same time; and forefoot strike (FFS) where the ball of the foot contacts the ground first. Footstrike angle boundaries for RFS-MFS and MFS-FFS were then set as the mean \pm one standard deviation of all footstrikes visually classified as MFS (as equivalently done by Altman and Davis [16] based on Strike Index). This resulted in boundaries of $10^{\circ}$ for RFS-MFS and $-7^{\circ}$ for MFS-FFS.

A probabilistic curve-clustering approach using a regression mixture model [20] was used to analyse the FSA data with the purpose of grouping participants based on those that showed similar trends in FSA with running velocity. This analysis was completed using the Curve Clustering toolbox [21] developed for Matlab (MathWorks Inc., Natick, MA, USA). The selection process for polynomial order (quadratic) and number of clusters (three) followed the recommendations of Gaffney [20]. The mean and standard deviation for each of the five footstrike variables for the participant data within each cluster were evaluated.

To determine the extent to which a reliable solution had been reached in the curve-clustering process an adapted bootstrapping approach was used [22]. Random resampling of the complete data set was conducted over sample sizes from 10 to 102 participant's data and the clustering process repeated in each case ( 25 repeat random samples for each sample size). The root mean square (RMS) difference between the random resampled and fully sampled cluster centre curves was evaluated as a measure of the reliability. 


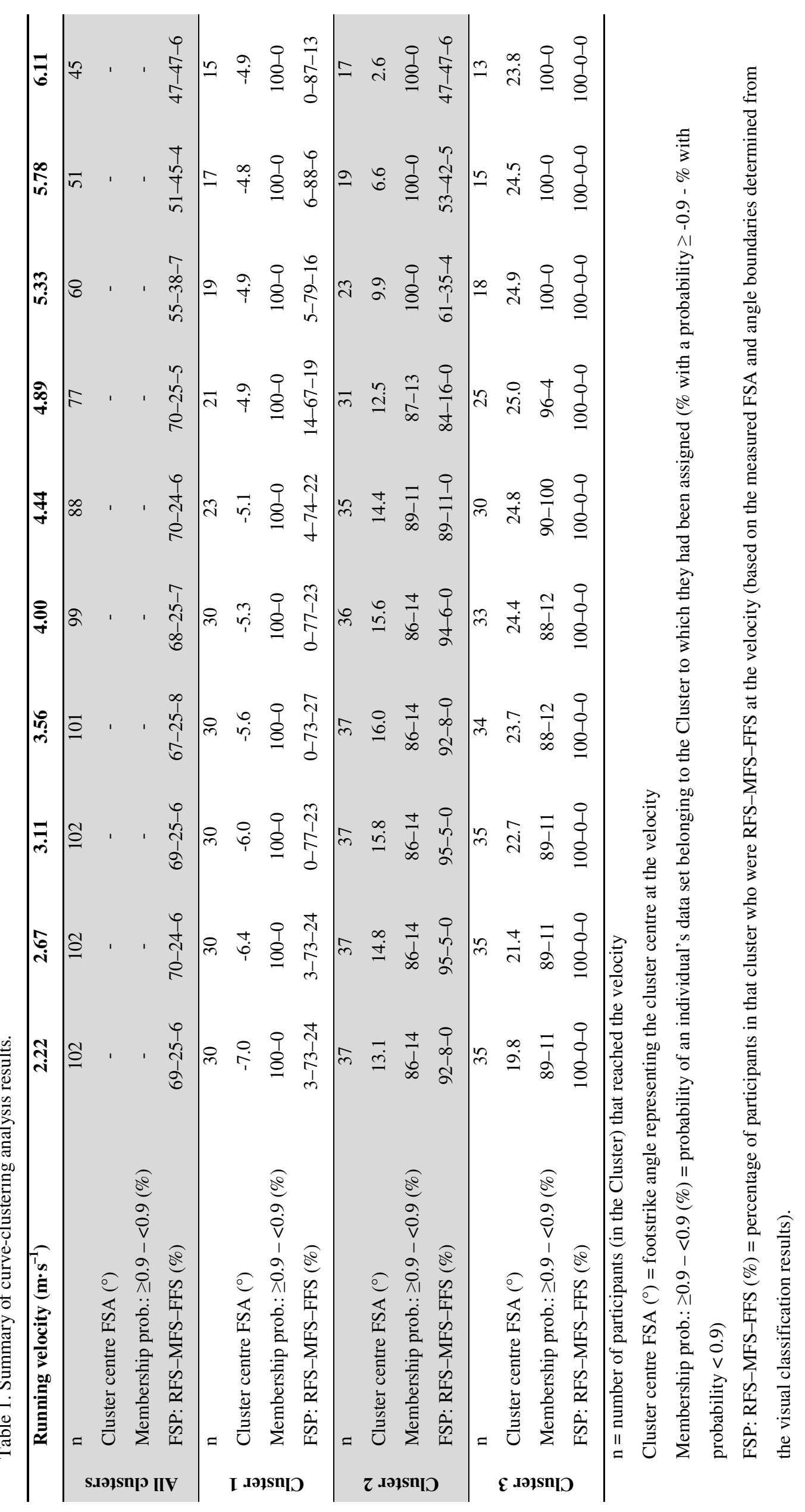




\subsection{RESULTS}

Of the 102 participants, 99 reached $4.0 \mathrm{~m} \cdot \mathrm{s}^{-1}$, thereafter there was a rapid drop off with only 59 participants reaching $5.3 \mathrm{~m} \cdot \mathrm{s}^{-1}$ and 45 completing all ten velocities (Table 1). The discrete FSP classification method indicated an overall trend away from RFS towards MFS and FFS at velocities $\geq 5.0 \mathrm{~m} \cdot \mathrm{s}^{-1}$. For velocities between $2.2-4.9 \mathrm{~m} \cdot \mathrm{s}^{-1}$ approximately $70 \%$ were RFS, $24 \%$ were MFS and $6 \%$ FFS, at $5.3 \mathrm{~m} \cdot \mathrm{s}^{-1}$ these percentages changed to 55\% RFS, 38\% MFS and 7\% FFS and at 6.1 $\mathrm{m} \cdot \mathrm{s}^{-1}$ they were $47 \%$ RFS, $47 \%$ MFS and $6 \%$ FFS.

The curve-clustering analysis indicated that the FSA - running velocity data were best represented by the following three clusters with an approximately equal number of participants in each (Figure 1 and Table 1):

- Cluster 1: small / negative FSA throughout $(\mathrm{n}=30)$

- Cluster 2: large positive FSA at low velocities, i.e. $\leq 4 \mathrm{~m} \cdot \mathrm{s}^{-1}$, transitioning to a smaller FSA at higher velocities, i.e. $\geq 5 \mathrm{~m} \cdot \mathrm{s}^{-1}(\mathrm{n}=37)$

- Cluster 3: large positive FSA throughout $(n=35)$

Typical footstrikes for each cluster are shown in Figure 2.

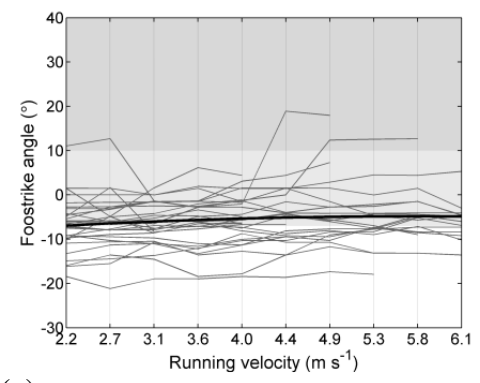

(a)

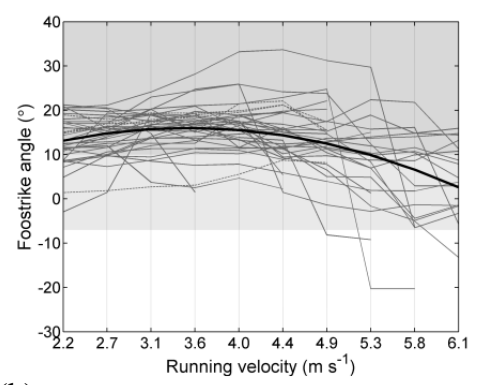

(b)

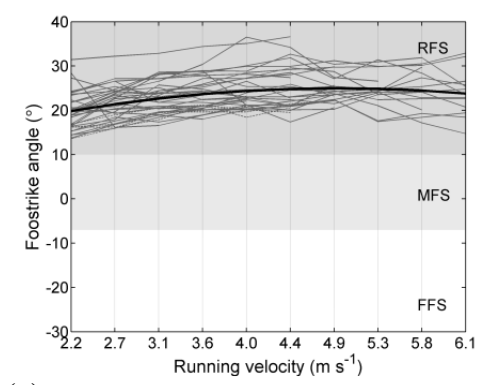

(c)

Figure 1. The three clusters of behaviour for FSA as a function of running velocity: (a) Cluster 1: small / negative FSA throughout; (b) Cluster 2: Large positive FSA at low velocities transitioning to a smaller FSA at higher velocities; and (c) Cluster 3: Large positive FSA throughout. In each plot the thick black line represents the cluster centre and the thin black lines the data for the individual participants within that cluster. Those participants with a low probability of cluster membership, i.e. probability $<0.9$, are shown as dashed lines. The three shaded regions on each plot approximately represent RFS (darkest), MFS (mid) and FFS (lightest). 
The curve-clustering reliability analysis indicated a rapid drop in RMS difference, to $<2^{\circ}$, between cluster centre curves for sample sizes up to 60 followed by a more gradual decrease thereafter (Figure 3(a)). This suggests that a reliable cluster analysis solution, representative of the broader population of recreational athletes, had been achieved using the 102 participants.

\section{Cluster 1}

\section{Cluster 2}

Cluster 3
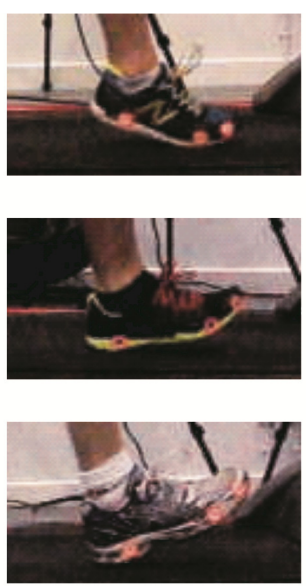

$2.7 \mathrm{~m} \cdot \mathrm{s}^{-1}$
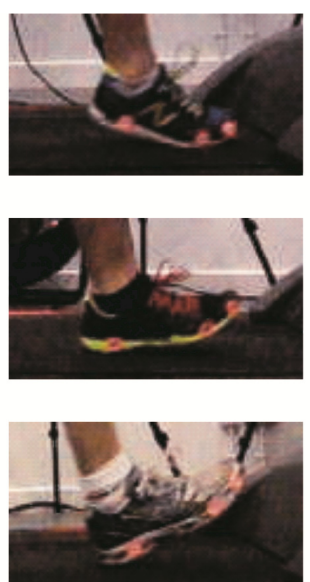

$4.0 \mathrm{~m} \cdot \mathrm{s}^{-1}$
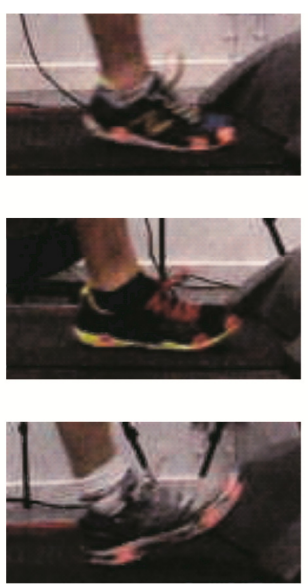

$5.8 \mathrm{~m} \cdot \mathrm{s}^{-1}$

Figure 2. Footstrikes for a typical participant in each cluster at low, intermediate and high running velocities.

The probability of an individual's data set belonging to the cluster to which they had been assigned provides another means of assessing confidence in the clustering process. (Figure 3 and Table 1). The probabilities were generally very close to one, with only five participants in Cluster 2 and four in Cluster 3 with membership probabilities $<0.9$ (Figure 3).

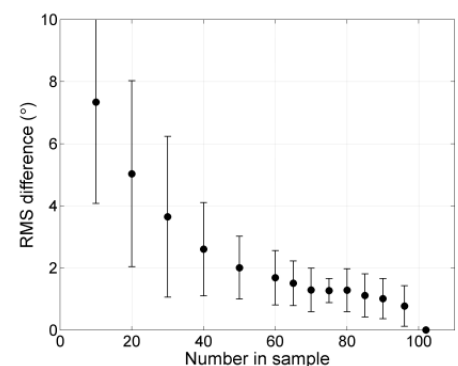

(a)

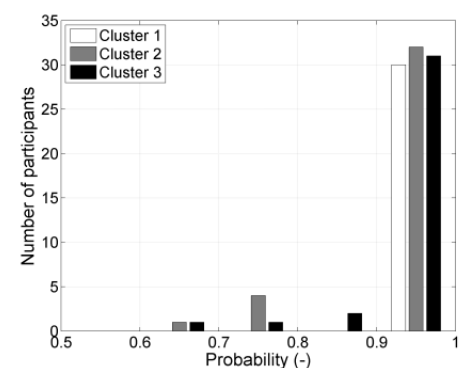

(b)

Figure 3. (a) Sample size versus RMS difference for the random resampling analysis. The $x$-axis represents the number of participants randomly selected, while the y-axis represents the RMS difference between the random resampled and fully sampled cluster centre curves. (b) Membership probability histogram for each cluster. 
The mean values for each cluster in footstrike angle, stride frequency, normalised stride length, ground contact time and duty factor are shown in Figure 4. The trends in FSA with running velocity, both within and between clusters, reflect the clustering results presented above. Both stride frequency and normalised stride length increased with increasing running velocity. Cluster 3 demonstrated lower stride frequencies and higher normalised stride lengths compared to Clusters 1 and 2. Notably, Cluster 2 values were closer to Cluster 1 values across all running velocities. Both ground contact time and duty factor decreased with increasing running velocity. Cluster 3 demonstrated higher ground contact times and duty factors compared to Cluster 1 . In this case, in contrast to stride frequency and normalised stride length, Cluster 2 tended to track Cluster 3 at lower velocities but shifted towards Cluster 1 at higher velocities, i.e. tracking the FSA results.

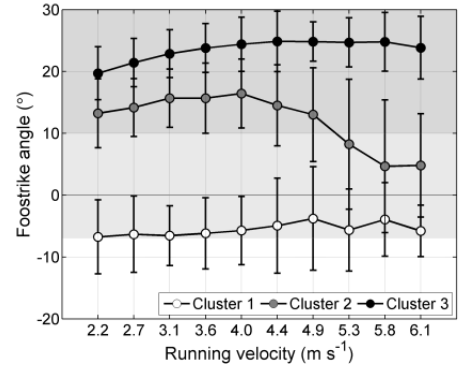

(a)

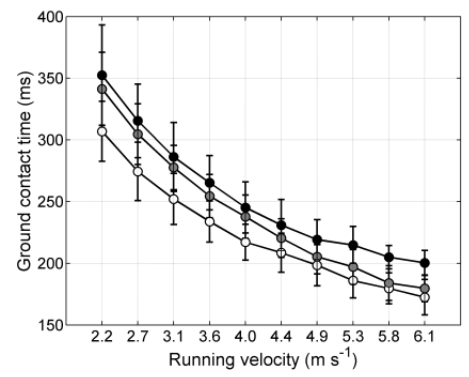

(d)

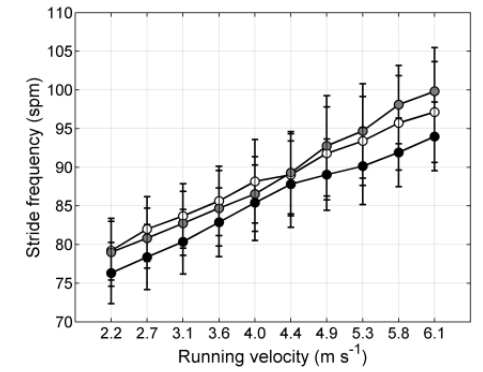

(b)

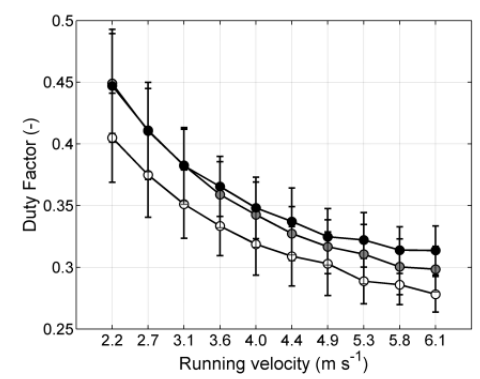

(e)

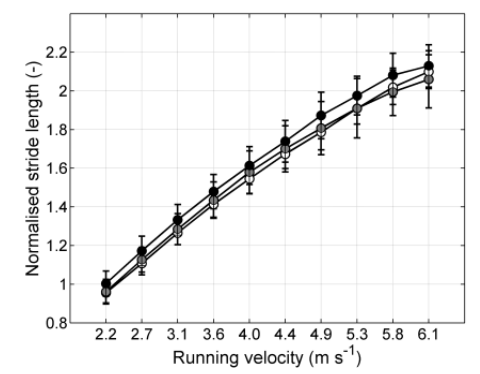

(c)

Figure 4. The effect of running velocity on (a) footstrike angle; (b) stride frequency; (c) normalised stride length, (d) ground contact time; and (e) duty factor for each of the three clusters. The data is shown as the mean and one standard deviation. The three shaded regions on plot (a) approximately represent RFS (darkest), MFS (mid) and FFS (lightest).

\subsection{DISCUSSION}


This study has quantified how FSA is affected by running velocity in recreational athletes. It was hypothesized that there would be an overall trend of reducing FSA, i.e. towards a more MFS / FFS, with increasing running velocity particularly above $5 \mathrm{~m} \cdot \mathrm{s}^{-1}$. The results indicated three distinct clusters of behaviour: Cluster 1 characterised by participants with a small / negative FSA throughout; Cluster 2 characterised by participants with a large positive FSA at low velocities $(\leq 4$ $\left.\mathrm{m} \cdot \mathrm{s}^{-1}\right)$ transitioning to a smaller FSA at higher velocities $\left(\geq 5 \mathrm{~m} \cdot \mathrm{s}^{-1}\right)$; and Cluster 3 characterised by participants with a large positive FSA throughout. Thus, the results were only in partial support of the hypothesis with only one of the three clusters (36\% of runners) showing a decrease in FSA with increasing running velocity.

For comparison to previous studies it is necessary to consider the discrete FSP classification results. Considering the whole group, the RFS-MFS-FFS percentages at running velocities below $5 \mathrm{~m} \cdot \mathrm{s}^{-1}$ were consistently around $70 \%-24 \%-6 \%$; thereafter, there was a substantial shift away from RFS towards MFS and FFS with the distribution being $47 \%-47 \%-6 \%$ at the highest velocity. This supports an overall group trend away from RFS towards MFS and FFS at running velocities above $5 \mathrm{~m} \cdot \mathrm{s}^{-1}$; however, of the 70 participants in the RFS category only nine converted to a MFS and one to a FFS, and of the 30 that reached the highest velocity 21 remained RFS throughout (see Supplementary Material for detailed numbers). These results are in good agreement with previous studies in terms of: the distribution of RFS-MFS-FFS runners at long-distance velocities [5-8]; the shift away from RFS towards MFS and FFS at velocities $\geq 5 \mathrm{~m} \cdot \mathrm{s}^{-1}[10,11]$; and partially with the distribution of RFS-MFS-FFS runners at middle-distance velocities [9].

The FSA boundaries between RFS-MFS and MFS-FFS were wider than those reported by Altman and Davis [16] $\left(10^{\circ}\right.$ and $-7^{\circ}$ here versus $8^{\circ}$ and $-2^{\circ}$ in [16]). The main reason for this is likely to be the differing methods used to define MFS runners from which the angle boundaries were set. This 
study used visual classification whereas [16] used Strike Index. Notably, in [16] although visual classification suggested the 60 shod trials to be equally split between RFS-MFS-FFS, i.e. 20-2020, when the same trials were analysed using Strike Index these numbers changed to 29-6-25. This suggests that more subjects tend to be classified as MFS through visual classification than through Strike Index; thus FSA boundaries are likely to be wider for the former, as found here. It is also worth noting that the difference was greater for the MFS-FFS boundary reinforcing the challenge in differentiating these footstrikes visually.

Curve-clustering analysis was considered the most appropriate means of investigating how recreational athletes adapt their FSA with running velocity. Clustering is often used as an initial data assessment method in order to group large amounts of data that display some inherent properties that lend themselves to natural groupings [20]. Therefore, it requires the data set to have natural groupings as opposed to being uniformly distributed, which was the case in the current study (a two-sample Kolmogorov-Smirnov test confirmed non-uniform distributions for all but the three highest velocities). This technique finds clusters that best describe the individual participants' data in an inclusive manner, i.e. it is robust in dealing with data sets of differing lengths, and requires no initial assumptions on how the participants might be best grouped. Thus, it addresses the purpose of this study whilst overcoming potential limitations of traditional statistical methods, e.g. $<50 \%$ of participants did not complete the full ten velocities.

The main limitation of curve-clustering analysis occurs if the clusters overlap, which can reduce the efficiency of the clustering process [20]. This may have occurred to some extent with Clusters 2 and 3, which displayed similar FSA values and trend over the lower velocities, potentially increasing the difficulty of clustering participants who failed to reach the higher velocities. This is reflected in the few lower membership probabilities for these two clusters (Figure 3). A solution 
would have been to neglect participants who failed to reach a velocities $>4 \mathrm{~m} \cdot \mathrm{s}^{-1}$; however, this only accounted for three participants.

The effect of running velocity on the remaining gait cycle variables of stride frequency (increased), normalised stride length (increased), ground contact time (decreased) and duty factor (decreased) were in good overall agreement with previous studies [23-27]. Furthermore, the difference in these variables between Clusters 1 and 3 were in agreement with studies comparing MFS / FFS and RFS runners $[5,9]$. Perhaps of greatest interest is the behaviour of Cluster 2 runners and, in particular, the relatively high stride frequencies and low stride lengths even at low velocities where FSAs were large positive, i.e. they tended to track Cluster 1 despite FSAs closer to Cluster 3. Given existing evidence for a positive relationship between stride length and impact loading, e.g. [28,29], Cluster 2 runners appear to adopt a stride length which may result in reduced impact loading compared to Cluster 3 runners.

All clusters demonstrated an underlying trend for FSA to increase very gradually with running velocity for velocities up to $5 \mathrm{~m} \cdot \mathrm{s}^{-1}$ as similarly reported in previous studies $[11,30]$. For Cluster 1 this was minimal at $\sim 2^{\circ}$ over the velocity range. For Cluster 2 this was only present up to $4 \mathrm{~m} \cdot \mathrm{s}^{-1}$ and was again small at $\sim 3^{\circ}$, before FSA started to reduce (as similarly reported in [11]). For Cluster 3 the increase was largest at $\sim 5^{\circ}$. This trend of increasing FSA for runners already exhibiting a large positive FSA is of some concern. It could be hypothesized that it was due to Cluster 3 runners increasing stride length through increasing hip range of motion without altering knee and ankle kinematics, which may have implications for over-striding. A first approximation to examine this hypothesis can be achieved based on simple trigonometry; by considering a fixed leg length at touchdown [30-32] and using the treadmill velocity and ground contact time to establish horizontal touchdown position to estimate FSA. This approximation predicted an increase in FSA very similar to that observed experimentally, to within $1^{\circ}$ for velocities up to $4.4 \mathrm{~m} \cdot \mathrm{s}^{-1}$; however, confirmation 
of the hypothesis would require further study. From a biomechanics perspective, as running velocity increases, touchdown thigh angle tends to become more horizontal which dominates smaller increases in knee flexion and negligible change in ankle angle [30]. This leads to touchdown extending further in-front of the body [31]. Cluster 2 appear to modulate these changes by reducing footstrike angle which, similarly to the lower stride length for these runners as discussed above, may result in reduced impact loading compared to Cluster 3 runners $([11,33])$. Although whether these technique changes represent a conscious effort by Cluster 2 runners to reduce impact loading is not currently known.

Cluster analysis is often used as an initial step of grouping data prior to further analysis. In this study it has provided an objective means of classifying the runners into one of three groups of behaviour, an efficient way of summarising the results from all 102 participants as well as a means to allow additional runners to be classified. The current approach may also help to explain previous FSA versus running velocity results and, in particular, the increased standard deviations at higher velocities $[11,30]$. Specifically these groups may not have been homogenous but have contained a mix of runners from the different Clusters identified here. Finally, this novel approach has identified some specific areas that warrant further investigation. Principally the biomechanical differences between Clusters 2 and 3 at low velocities (where FSAs are similar) versus high velocities (where FSAs differ) in order to better understand how the observed behaviours are achieved and any implications these may have for performance and / or injury.

The main limitations of this study were the fixed velocity range used for all participants and the lack of counterbalancing of these velocities. Ideally subject-specific velocities would have been used spanning an individual's walk-run transition to maximum sprint velocity. This approach was infeasible based on the number of participants recruited as it would have required two sessions per participant, the first to establish these limiting velocities. Furthermore, maximal sprint velocity 
would have been challenging to accurately determine given the recreational athlete population some of whom were un-used to and / or uncomfortable running at high velocities. The fixed velocities approach also allowed the results to be considered in the context of comparable previous studies as presented herein $[11,30]$. Thus, although the current data lacks a subject-specific velocity scaling, this is unlikely to have influenced the main outcomes of the curve-clustering analysis. The threat of fatigue influencing the results due to the lack of counterbalancing was considered small. All participants were encouraged to use the two minute rests between velocities, only the final two or three velocities pushed the participant beyond their comfortable running range and all were engaged in regular exercise that included running.

In summary, using FSA to quantify FSP has allowed the changes with velocity to be examined with greater resolution than previous studies that relied on discrete classification. The curve-clustering analysis on the FSA - running velocity data provided a novel and relevant means of grouping participants for further assessment of their running biomechanics. Three distinct patterns of FSA velocity behaviour were identified: small / negative FSA throughout; large positive FSA at low velocities transitioning to a smaller FSA at higher velocities; and large positive FSA throughout. Notably, there were two trends in behaviour for runners with large positive FSAs at lower velocities, only one of which demonstrated a decrease in FSA with increasing running velocity.

\section{Conflict of interest statement}

The authors have no conflict of interest. 


\section{References}

[1] Daoud AI, Geissler GJ, Wang F, Saretsky J, Daoud YA, Lieberman DE. Foot Strike and Injury Rates in Endurance Runners: A Retrospective Study. Med Sci Sport Exerc 2012;44:1325-34.

[2] Diebal AR, Gregory R, Alitz C, Gerber JP. Forefoot Running Improves Pain and Disability Associated With Chronic Exertional Compartment Syndrome. Am J Sports Med 2012;40:1060-7.

[3] Laughton CA, McClay Davis I, Hamill J. Effect of Strike Pattern and Orthotic Intervention on Tibial Shock During Running. J Appl Biomech 2003:19;153-68.

[4] Nunns M, House C, Fallowfield J, Allsopp A, Dixon S. Biomechanical characteristics of barefoot footstrike modalities. J Biomech 2013;46:2603-10.

[5] Hasegawa H, Yamauchi T, Kraemer WJ. Foot Strike Patterns of Runners at the $15 \mathrm{~km}$ point during an Elite-level Half Marathon. J Strength Cond Res 2007;21:888-93.

[6] Bertelsen ML, Jensen JF, Nielsen MH, Nielsen RO, Rasmussen S. Footstrike patterns among novice runners wearing a conventional, neutral running shoe. Gait Posture 2013;38:354-6.

[7] Larson P, Higgins E, Kaminski J, Decker T, Preble J, Lyons D, et al. Foot strike patterns of recreational and sub-elite runners in a long-distance road race. J Sport Sci 2011;29:1665-73.

[8] Kerr BA, Beauchamp L, Fisher V, Neil R. Footstrike patterns in distance running. In: Nigg, BM, Kerr BA, editors. Biomechanical Aspects of Sports Shoes and Playing Surfaces, Calgary, Canada: University Printing; 1983, p. 135-42.

[9] Hayes P, Caplin N. Foot strike patterns and ground contact times during high-calibre middledistance races. J Sport Sci 2012;30:1275-83.

[10] Keller TS, Weisberger AM, Ray JL, Hasan SS, Shiavi RG, Spengler DM. Relationship between vertical ground reaction force and speed during walking, slow jogging and running. Clin Biomech 1996;11:253-9. 
[11] Nigg BM, Bahlsen HA, Luethi SM, Stokes S. The influence of running velocity and midsole hardness on external impact forces in heel-toe running. J Biomech 1987;20:951-9.

[12] van Gent RM, Siem D, van Middlekoop M, van Os AG, Bierma-Zeinstra AMA, Koes BW. Incidence and determinants of lower extremity running injuries in long distance runners: a systematic review. Brit J Sport Med 2007;41:469-80.

[13] Cavanagh PR, Lafortune MA. Ground Reaction Forces in Distance Running. J Biomech $1980 ; 13: 397-406$.

[14] McCaw ST, DeVita P. Errors in alignment of center of pressure and foot coordinates affect predicted lower extremity torques. J Biomech 1995;28:985-8.

[15] Chockalingam N, Giakas G, Iossifidou A. Do strain gauge force platforms need in situ correction? Gait Posture 2002;16:233-7.

[16] Altman AR, Davis IS. A kinematic method for footstrike pattern detection in barefoot and shod runners. Gait Posture 2012;35:298-300.

[17] Kernozek TW, Ricard MD. Foot placement angle and arch type: effect on rearfoot motion. Arch Phys Med Rehab 1990;71:988-91.

[18] Sih BL, Hubbard M, Williams KR. Correcting out-of-plane errors in two-dimensional imaging using nonimage-related information. J Biomech 2001;34:257-60.

[19] Kram R, Domingo A, Ferris DP. Effect of reduced gravity on the preferred walk-run transition speed. J Exp Biol 1997;200:821-6.

[20] Gaffney SJ. Probabilistic Curve-Aligned Clustering and Prediction with Regression Mixture Models. Chapter 3: Curve Clustering with Regression Mixtures. Irvine, CA: University of California; 2004 ( $\mathrm{PhD}$ thesis).

[21] CCToolbox. Available at: http://www.datalab.uci.edu/resources/CCT/

[22] Loong CKP, Zhou H-X, Chase PB. Persistence Length of Human Cardiac $\alpha$-Tropomyosin Measured by Single Molecule Direct Probe Microscopy. PLoS ONE:7;e39676. 
[23] Padulo J, Annino G, Migliaccio GM, D’ottavio S, Tihanyi J. Kinematics of Running at Different Slopes and Speeds. J Strength Cond Res 2012;26:1331-9.

[24] Brughelli M, Cronin J, Chaouachi A. Effects of running velocity on running kinetics and kinematics. J Strength Cond Res 2011;25:933-9.

[25] Salvidge C, Hayes P. Changes in muscle tendon unit stiffness with increasing running speed. Brit J Sport Med 2010;44:i27.

[26] Weyand PG, Sternlight DB, Bellizzi MJ, Wright S. Faster top running speeds are achieved with greater ground forces not more rapid leg movements. J Appl Physiol 2000;89:1991-9.

[27] Minetti AE. A model equation for the prediction of mechanical internal work of terrestrial locomotion. J Biomech 1998;31:463-8.

[28] Heiderscheit BC, Chumanov ES, Michalski MP, Wille CM, Ryan MB. Effects of Step Rate Manipulation on Joint Mechanics during Running. Med Sci Sport Exerc 2011;43:296-302.

[29] Schubert AG, Kempf J, Heiderscheit BC. Influence of Stride Frequency and Length on Running Mechanics: A Systematic Review. Sports Health 2014:6;210-7.

[30] De Wit B, De Clercq D, Aerts P. Biomechanical analysis of the stance phase during barefoot and shod running. J Biomech 2000:33;269-78.

[31] Nilsson J, Thorstensson A, Halbertsma J. Changes in leg movement and muscle activity with speed of locomotion and mode of progression in humans. Acta Physiol Scand 1985:123;45775.

[32] Tsatalas T, Giakas G, Spyropoulos G, Sideris V, Lazaridis S, Kotzamanidis C, et al. The effects of eccentric exercise-induced muscle damage on running kinematics at different speeds. J Sport Sci 2013:31;288-98.

[33] Gerritson GM, van den Bogert AJ, Nigg BM. Direct dynamics simulation of the impact phase in heel-toe running. J Biomech 1995:28;661-8. 


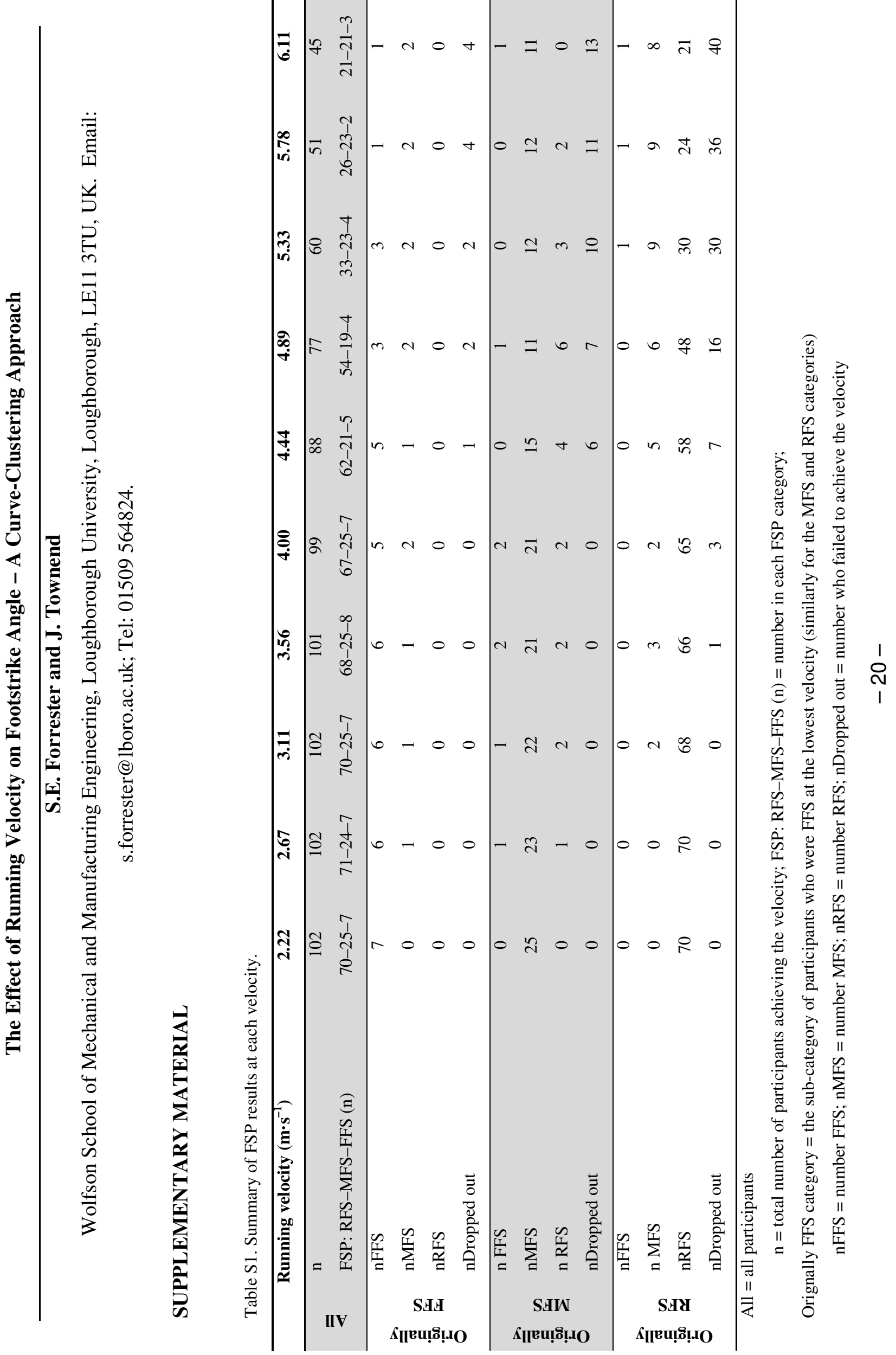

